\title{
Fomentando el trabajo autónomo y cooperativo en un contexto de cooperación internacional: VI Programa Intensivo sobre el futuro de la banca y las finanzas \\ Promoting autonomous and cooperative work in the context of international cooperation: VI Intensive Program in future of banking and finance
}

\author{
Pedro Fernández Sánchez ${ }^{1}$, Elizabeth Frank ${ }^{2}$ \\ fersan.fcee@ceu.es, elizabeth.frank@ceu.es \\ ${ }^{1}$ Economía \\ Universidad CEU San Pablo \\ Madrid, España \\ ${ }^{2}$ Empresa \\ Universidad CEU San Pablo \\ Madrid, España
}

\begin{abstract}
Resumen- Enmarcado dentro del compromiso de la universidad española de formar no sólo en conocimientos sino también en competencias, este trabajo presenta una nueva forma de enfocar la elaboración de los trabajos de fin de grado. En esta propuesta prima la adquisición de competencias transversales por parte del estudiante, combinando el trabajo autónomo con el cooperativo gracias a la colaboración entre varias universidades europeas. A lo largo de su último curso de grado, los estudiantes, guiados por varios profesores, deben investigar sobre varias cuestiones relacionadas con el mundo de la banca, de las finanzas y de la ética, para prepararse de cara al encuentro. Esta preparación previa les permite abordar durante la semana en que tiene lugar el programa intensivo y junto con el resto de compañeros europeos, la elaboración de un trabajo académico desde una perspectiva internacional. Esta investigación, además, les puede servir de base para la posterior elaboración y defensa de su trabajo de fin de grado en su universidad de origen.
\end{abstract}

Palabras clave: Trabajo de fin de grado, trabajo autónomo, trabajo cooperativo, competencias transversales, internacionalización.

Abstract- It is the objective of every Spanish University to not merely provide theoretical knowledge and education, but also to develop student's competences and skills. The project presented in this paper presents a new, innovative approach for students and professors to develop and redact Bachelors' final degree projects. Providing participating students and professors the possibility to acquire and improve transversal key competences is the main priority of the project. An ample range of skills, such as language, social, digital and professional skills amongst others, will be developed, placing special emphasis on combining autonomous and cooperative work with the participants of several European universities. During their final year Bachelor students, under the supervision of their professors, investigate several topics related to the world of banking, finance and ethics. This investigation prepares them for the intensive week, where they meet their European counterparts from the other participating European Universities. Placed in "Topic Teams" they have to write a scientific paper and present it during the week. This paper could potentially be the basis for their individual Bachelor's final degree project at their Home Institution.
Keywords: Bachelor's final degree project, autonomous work, cooperative work, transversal competences, internationalization.

\section{INTRODUCCIÓN}

Como miembros de la comunidad universitaria debemos dar respuesta a las necesidades de la sociedad. Para ello, y tal y como se señala en la Declaración Mundial sobre la Educación Superior en el siglo XXI, tenemos como misión educar, formar e investigar (Cátedra UNESCO, 2000).

Es por ello por lo que la elaboración de los trabajos de fin de grado debe constituir una de las piedras angulares en la formación de nuestros estudiantes. Partiendo de este enfoque, la Universidad CEU San Pablo lleva colaborando desde hace seis años con 5 universidades europeas en el desarrollo de un programa intensivo en el que los estudiantes, en una semana y tras una labor previa en los meses anteriores al encuentro, deben desarrollar un trabajo de investigación y presentarlo ante el resto de compañeros y profesores. Esto permite a los participantes no sólo realizar un trabajo académico, sino también adquirir una serie de competencias transversales que les resultarán muy valiosas para su posterior desarrollo profesional.

De esta forma la experiencia docente que se presenta permite una formación integral del estudiante, que incluye, y de ahí lo novedoso, el trabajo en un entorno multicultural con todo lo que ello implica. También incide en el trabajo autónomo del alumno, previo a la celebración del programa intensivo, así como en el trabajo cooperativo, que tiene lugar durante la semana en la que se celebra el programa.

El presente trabajo se ha estructurado en cuatro partes además de las conclusiones. En primer lugar se explica por qué resulta interesante poner en marcha una iniciativa de este tipo. A continuación se describe en qué consiste el programa, así como qué tipos de actividades se desarrollan a lo largo del mismo. En último término $\mathrm{y}$ antes de las conclusiones se 
exponen los resultados que se han obtenido en base a las encuestas que realizan los alumnos participantes.

\section{CONTEXTO}

En la actualidad la práctica totalidad de los grados incluyen en el último curso la elaboración de un trabajo de fin de grado. Constituye una de las asignaturas más importantes dentro del currículo universitario, en la medida en que supone la culminación de los estudios de grado y debe reflejar la madurez adquirida por el estudiante a lo largo de su aprendizaje universitario. En este trabajo se presenta una nueva forma de enfocar la elaboración de este tipo de trabajos.

\section{A. Objetivos}

El programa intensivo pretende ir más allá de la realización de un trabajo de investigación tradicional. Procura, en la línea de lo que defiende Fernández Batanero (2004, p.5), crear una experiencia en los estudiantes, en la que asocien la idea de aprendizaje a las de participación, búsqueda de información y trabajo autónomo y cooperativo para su posterior discusión, todo ello combinado con el empleo de nuevos recursos. Además, se persigue que los participantes adquieran las competencias transversales más demandadas por los empleadores, según la escala desarrollada por Martínez Clares y González Morga (2019, p.9). Competencias que les permitan un mejor desenvolvimiento en el mundo laboral al que se incorporarán casi inmediatamente.

En el caso que aquí se presenta los estudiantes deben desarrollar durante un período de 3 a 4 meses unos trabajos académicos sobre el ámbito temático del encuentro. Han de trabajar de manera autónoma y conjuntamente, tanto de forma presencial como remota, en documentos académicos interuniversitarios específicos, que deben entregar y presentar ante el resto de sus compañeros y profesores en la universidad elegida como sede para la realización del encuentro. Este programa exige conocimientos previos sobre las materias objeto de análisis, y permite a los participantes desarrollar una amplia serie de habilidades como el dominio del idioma inglés, la capacidad de exponer en público en esa lengua, el trabajo en equipo internacional o el desarrollo de habilidades de trabajo colaborativo.

Constituye un claro ejemplo de innovación docente en el ámbito de los trabajos de fin de grado, pues permite a los estudiantes de la Universidad CEU San Pablo presentar y defender la investigación realizada durante el programa intensivo.

Supone además un esfuerzo de cooperación internacional en docencia, ya que en el programa participan profesores de varias universidades europeas que imparten seminarios teóricos y prácticos a los alumnos y les guían en la elaboración de un documento científico. En concreto, las Universidades que asistieron al VI encuentro fueron Inholland University of Applied Sciences (Países Bajos) como coordinadora del proyecto, Universidad CEU San Pablo (España), University College Ghent (Bélgica), University of Finance and Administration in Prague (República Checa), Higher School of Economics (Rusia) con estatuto de colaborador especial, y la
Universidad de Zaragoza (España) como organizadora en el año 2019 del programa intensivo.

\section{B. Público Objetivo}

Alumnos de último curso matriculados en la asignatura de trabajo de fin de grado de cualquier titulación de la Facultad de Ciencias Económicas y Empresariales. En el caso de la Universidad CEU San Pablo y dado que únicamente hay cinco plazas, se contacta con todos los estudiantes con una nota media superior a 7. El proceso de selección es muy exigente, debiendo cumplir los aspirantes varios requisitos tales como un nivel de inglés adecuado, excelencia académica y una carta de motivación. Además, han de participar en una dinámica de grupo con la que se valora su capacidad para trabajar en equipo. Se les puntúa en cada uno de los apartados y se establece un ranking a partir del cual se les ofrece participar en el programa.

\section{DESCRIPCIÓN}

El programa intensivo se desarrolla a lo largo de una semana en una de las universidades participantes, generalmente en el mes de abril. El último encuentro tuvo lugar en Zaragoza entre el 7 y 13 de abril de 2019. Sin embargo, los estudiantes deben comenzar a trabajar desde diciembre, guiados por los profesores de sus respectivas universidades, así como por los responsables de cada uno de los temas o "topics" en los que el programa se organiza. Desde el mismo momento en que acceden al programa se les entregan unas credenciales de Moodle para que tengan acceso a toda la información del programa, incluido el "Student Handbook" o guía del estudiante, donde se explican todos los aspectos del mismo. En esa cuenta es donde deben ir subiendo los trabajos en las fechas previstas.

Concretamente los estudiantes han de elaborar tres trabajos a lo largo del programa:

1.- Un "national paper", en el que explican la evolución de sus respectivas economías desde el año 2008. Elaborado conjuntamente por todos los estudiantes de cada país, el objetivo es poner en contexto los posteriores trabajos temáticos y dar a conocer a los participantes del resto de países los problemas a los que se han enfrentado las economías nacionales a raíz de la gran recesión. Este trabajo obliga a los alumnos de cada país a buscar información, organizarla, presentarla dando una estructura de informe y posteriormente exponerla ante sus compañeros en forma de póster el primer día del encuentro.

2.- Un "trabajo individual" en función del tema escogido por cada alumno. En concreto, el programa se configura en torno a seis "topics" o temas:

a. Business rating (rating empresarial)

b. Taxation and fiscal regulation (imposición y regulación fiscal)

c. Corporate governance (gobierno corporativo)

d. Banking 2020 and beyond (el futuro de la banca más allá del horizonte 2020)

e. Alternative ways of financing of small and mediumsized enterprises (sistemas alternativos de financiación de pequeñas y medianas empresas)

f. Social responsible investment (inversiones socialmente responsables) 
De los 6 temas propuestos, cada estudiante escoge uno diferente y, a partir de las guías fijadas en la guía del estudiante, han de responder a una serie de cuestiones. El objetivo de este trabajo previo, que comienza en el mes de diciembre, es que los alumnos adquieran tanto los conocimientos teóricos necesarios sobre el tema objeto de estudio, como que manejen información sobre la situación específica de su país. A mediados de marzo los estudiantes deben haber finalizado este trabajo individual, y han de publicarlo en la plataforma.

En esta fase se combina el trabajo autónomo del estudiante con la guía de dos profesores que dirigen cada tema. Además, y para que los estudiantes se vayan familiarizando con el resto de compañeros que participarán en el mismo tema, se crean grupos de WhatsApp, gestionados y dirigidos por los responsables del "topic". A través de los mismos los alumnos se presentan al resto de compañeros, mediante videos y fotos, describiendo sus aficiones, cómo es su vida universitaria, etc. Esta herramienta permite a los profesores guiar a los estudiantes mientras responden a las preguntas planteadas para cada tema. A finales de marzo se establece una reunión vía Skype para evaluar la marcha del trabajo individual y preparar el trabajo del grupo de cara al encuentro.

3.- Un "international paper" que se realiza in situ durante la semana que tiene lugar el programa intensivo. A lo largo de cuatro días, los grupos internacionales elaboran un informe académico sobre el tema escogido. Guiados por los profesores responsables, y a través de herramientas como Google Docs que permiten trabajar en línea, los estudiantes han de dar estructura al trabajo, fijar los objetivos del mismo, desarrollar el tema y extraer conclusiones. Cada estudiante aporta el conocimiento en detalle de lo ocurrido en su país de origen, y lo pone en común con el resto de compañeros, tratando de establecer pautas comunes de comportamiento o poniendo de relieve las diferencias existentes entre sus países de origen. Mientras los estudiantes trabajan, los profesores guían, revisan el documento y realizan los comentarios que consideran oportunos en tiempo real. Realizan además tutorías teóricas y prácticas.

El día previo a la clausura los trabajos son corregidos por los profesores, que evalúan los aspectos formales y contenidos de los mismos. En el último encuentro cada trabajo fue revisado por tres profesores, siguiendo un esquema de evaluación previamente fijado por la organización. Mientras se realizaba la evaluación, los estudiantes trabajaban en la elaboración de una presentación, que fue defendida el día de la clausura (y que también fue evaluada por los profesores). Para una valoración más objetiva los profesores emplean dos rúbricas. Una se utiliza para el trabajo escrito, que supone un $80 \%$ de la valoración final, y la otra para la presentación oral, que supone el $20 \%$ restante. Estas rúbricas tienen en cuenta aspectos tales como la capacidad de cada grupo para plantear hipótesis y analizar problemas, la estructura del trabajo, el talento para proponer soluciones y recomendaciones o consejos concretos y útiles, la capacidad del grupo para conectar con el público en la presentación oral, el ser capaz de realizar la presentación en el tiempo establecido, el empleo de un lenguaje claro, el mantener una actitud tranquila o la capacidad para generar un debate en la audiencia una vez finalizada la presentación.

Como ya se ha explicado en el caso de la Universidad CEU San Pablo los trabajos realizados sirvieron de base a los estudiantes para la elaboración y defensa de sus respectivos trabajos de fin de grado. El programa además está diseñado para que pueda ser convalidado como una materia optativa de 3 créditos ECTS.

El programa intensivo pretende hacer de la elaboración del trabajo de fin de grado una experiencia para el estudiante. El trabajo desarrollado en los meses anteriores sirve de base para el que se tendrá lugar en la semana del encuentro. A lo largo de la misma los estudiantes no sólo son guiados por los profesores en los diferentes temas, sino que también asisten a talleres interculturales y conferencias. El objetivo último como ya se comentó es la adquisición de habilidades transversales demandadas por las empresas y que les permitirán estar mejor preparados de cara a su incorporación al mercado laboral.

Aunque el programa se desarrolla a lo largo de una semana, requiere de una preparación previa, tal y como se ha explicado. Durante varios meses los estudiantes aprenden el valor del trabajo autónomo y cooperativo, pues deben realizar trabajos con sus compañeros (paper nacional e internacional), así como por su propia cuenta (el trabajo individual). En la Tabla 1 se han resumido las etapas en las que se divide el programa que culminan con la celebración de la semana del encuentro.

\section{Tabla 1}

Cronología de las actividades desarrolladas a lo largo del programa intensivo.

\begin{tabular}{|c|l|}
\hline Fecha & \multicolumn{1}{|c|}{ Relación de actividades } \\
\hline $\begin{array}{c}\text { Septiembre } \\
\text { de } 2018\end{array}$ & $\begin{array}{l}\text { Reunión preparatoria de los profesores. } \\
\text { Revisión de la guía del estudiante e } \\
\text { incorporación de las mejoras propuestas al } \\
\text { finalizar el encuentro anterior. }\end{array}$ \\
\hline $\begin{array}{c}\text { Octubre de } \\
2018\end{array}$ & $\begin{array}{l}\text { Proceso de selección de los estudiantes que } \\
\text { participarán por cada universidad. }\end{array}$ \\
\hline $\begin{array}{c}1 \text { de } \\
\text { de } 2018 \text { al } \\
1 \text { de marzo } \\
\text { de } 2018\end{array}$ & $\begin{array}{l}\text { Elaboración del "national paper". Fecha límite } \\
\text { En paralelo comienza el trabajo autónomo: el } \\
\text { estudiante debe revisar la bibliografía de su } \\
\text { tema e investigar sobre el mismo. }\end{array}$ \\
\hline $\begin{array}{c}1 \text { al } 22 \text { de } \\
\text { marzo de } \\
2019\end{array}$ & $\begin{array}{l}\text { Creación de los grupos de WhatsApp. } \\
\text { El 22 de marzo es la fecha límite para que los } \\
\text { estudiantes publiquen su trabajo individual, } \\
\text { que comenzaron en diciembre, a la plataforma. }\end{array}$ \\
\hline $\begin{array}{c}\text { al } 13 \text { de } \\
\text { abril de } \\
2019\end{array}$ & $\begin{array}{l}\text { Semana del programa intensivo en Zaragoza. } \\
\text { Elaboración del paper internacional y } \\
\text { presentación. } \\
\text { Realización de actividades y talleres } \\
\text { relacionados con el programa. }\end{array}$ \\
\hline
\end{tabular}

Para concluir, señalar que el programa intensivo es posible gracias al trabajo y dedicación de los profesores que han participado en el mismo a lo largo de los últimos 7 años. Aunque no existe una dotación económica específica, los profesores pueden desplazarse gracias a la financiación que otorga la Comisión Europea a través del programa Erasmus+ 
Teaching Staff Mobility Programme. Por otra parte, los estudiantes reciben distintos tipos de ayudas de su universidad de origen para cubrir parte de sus gastos. Cabe señalar que el último encuentro, al tener lugar en España, no permitía a los profesores de la Universidad CEU San Pablo tener acceso a la financiación europea. En este caso el desplazamiento y la estancia fue sufragada íntegramente por la Facultad de Ciencias Económicas y Empresariales. Además, la Universidad CEU San Pablo becó con 180 euros a cada uno de los cinco estudiantes participantes en el encuentro de Zaragoza.

\section{Resultados}

¿Se han alcanzado los objetivos establecidos cuando se abordó la posibilidad de realizar una experiencia docente y de aprendizaje de este tipo? O lo que es lo mismo ¿los estudiantes consiguen adquirir unas competencias adicionales que no se obtienen cuando realizan un trabajo de fin de grado según los parámetros tradicionales?

Desde un punto de vista teórico se pretende que los participantes adquieran una serie de competencias transversales, que, de acuerdo con distintos informes internacionales, son consideradas fundamentales por las empresas a las que se incorporarán cuando finalicen sus estudios de grado (OIT, 2017 y CEDEFOP, 2014).

Siguiendo el esquema propuesto por Martínez Clares y González Morga (2019, p. 9), el programa se encuentra diseñado para que los participantes adquieran las principales competencias transversales, a saber:

\section{1.- Competencias instrumentales}

a) Organización y planificación: en los meses previos al programa intensivo y de acuerdo con el calendario de entregas y durante la semana de celebración del programa, los estudiantes han de organizar el trabajo y coordinarse entre ellos.

b) Comunicación oral y escrita: mediante la elaboración de tres trabajos escritos (uno con compañeros de su propia universidad, otro individual y otro con los estudiantes de otros países) y la presentación oral de dos de ellos (el "national paper" y el "international paper").

c) Uso de las tecnologías de la información y comunicación (TIC): reuniones vía Skype, grupos temáticos de WhatsApp, utilización de bases de datos, Moodle, Google docs.

d) Comunicación lengua extranjera: todo el programa se desarrolla en inglés con estudiantes de diferentes universidades europeas.

e) Toma de decisiones: sobre todo durante la semana del programa deben planificar el trabajo, decidir cómo va a ser la estructura del paper, así como qué información es o no es relevante (ya que existe un límite a la extensión de los trabajos).

f) Diseño y gestión de proyectos profesionales y de vida y toma de decisiones: el programa refuerza el enfoque metodológico para planificar, orientar y dirigir los procesos del proyecto, con la perspectiva de mejorar la futura inserción laboral de los participantes. g) Gestión de la información y del conocimiento: los alumnos aprenden a procesar y sintetizar la información para su empleo con un enfoque crítico.

\section{2.- Competencias Personales:}

a) Trabajo en equipo: a lo largo del programa en dos ocasiones, para la elaboración del paper nacional con los compañeros de su propia universidad, así como para la elaboración del trabajo sobre su tema con los estudiantes europeos que forman parte de su grupo. Además, este trabajo en equipo tiene lugar tanto presencialmente (por ejemplo, en la semana del encuentro), como remotamente (en los meses previos al mismo).

b) Interacción social: el programa está diseñado para que esa interacción comience en el momento en que se crea el grupo de WhatsApp, continúe durante la celebración de la semana intensiva, y prosiga más allá de su finalización.

c) Compromiso ético profesional y responsabilidad social: todo el programa está diseñado a partir de la premisa de qué falló en la gran recesión y de la importancia de la ética en los procesos de toma de decisiones en las empresas.

d) Control emocional: necesario cuando se trabaja bajo presión ya que hay que elaborar informes con fechas de entrega muy ajustadas. Incluye la resolución de conflictos, que aparecen cuando varias personas trabajan en equipo y no tienen por qué tener los mismos puntos de vista.

\section{3.- Competencias sistémicas}

a) Trabajo autónomo: al estudiante se le facilita material y unas cuestiones y debe ir trabajando sobre las mismas. De igual manera cuando comienza a trabajar en equipo, si bien cuenta con apoyo de profesores, debe tomar decisiones.

b) Actitud emprendedora: se trata de estudiantes proactivos y el programa busca esta competencia en ellos. Ellos han de ser autónomos y decidir, limitándose el papel del profesor al de guía.

c) Creatividad e innovación: estas competencias se ven plasmadas en los videos que realizan, en las presentaciones, en la elaboración del póster, etc.

d) Adaptación: los alumnos se han de adaptar a trabajar en un equipo internacional. Han de tener en cuenta las diferencias culturales que pueden existir o la barrera del idioma, y ser capaces de superarlos.

e) Motivación: por conocer, por investigar, por poder participar en un proyecto internacional y conocer a otros estudiantes y trabajar con ellos.

f) Investigación: todo el programa gira en torno a la misma.

Aunque resulta complicado valorar la adquisición de todas estas competencias, el programa trata de hacerlo a través, por un lado, de la evaluación del trabajo internacional elaborado por cada uno de los grupos de estudiantes y de su presentación oral. Para ello, y como se ha comentado anteriormente, se emplean dos rúbricas. Con ellas, si bien se evalúa objetivamente la adquisición de varias de las competencias mencionadas (como la comunicación oral y escrita, la creatividad, o la investigación), en el caso de otras competencias su valoración 
se realiza de manera indirecta. Es el caso, por ejemplo, de la competencia del trabajo en equipo. Es indudable que los estudiantes cooperan, y esa cooperación es evaluada indirectamente a través de estas rúbricas, ya que cuanto mejor sea dicho trabajo en equipo mejor resultará el trabajo académico valorado por los profesores.

Por otro lado se evalúa la adquisición de estas competencias transversales preguntándoles a los propios estudiantes. Al finalizar el programa han de realizar una encuesta anónima en la que se les preguntan diferentes cuestiones relacionadas con el mismo. A continuación, se presentan los resultados de estas encuestas para los dos últimos años académicos: 2018 (Praga) y 2019 (Zaragoza). La razón por la que se muestran sólo los dos últimos a pesar de haber tenido lugar seis encuentros, reside en que el programa se reestructuró ligeramente a partir del cuarto encuentro. De los resultados se desprende tal y como se puede observar en la Tabla 2, que los estudiantes valoran muy positivamente las competencias adquiridas al finalizar el programa:

\section{Tabla 2}

Evaluación general del IP por parte de los estudiantes en las dos últimas ediciones (escala de 1 a 5 )

\begin{tabular}{|l|c|c|}
\hline Cuestiones & 2018 & 2019 \\
\hline $\begin{array}{l}\text { Desde un punto de vista académico el } \\
\text { programa intensivo ha aumentado mis } \\
\text { conocimientos }\end{array}$ & 4 & 4 \\
\hline $\begin{array}{l}\text { El programa intensivo me ha permitido } \\
\text { mejorar mis habilidades personales }\end{array}$ & 4,7 & 4 \\
\hline $\begin{array}{l}\text { El programa intensivo me ayudará en mi } \\
\text { futura carrera profesional }\end{array}$ & 4,6 & 4,2 \\
\hline $\begin{array}{l}\text { El programa intensivo ha mejorado mis } \\
\text { competencias interculturales }\end{array}$ & - & 4,2 \\
\hline $\begin{array}{l}\text { Los profesores han sabido enseñarme y } \\
\text { guiarme a lo largo del programa }\end{array}$ & 4,1 & 4,5 \\
\hline $\begin{array}{l}\text { El WhatsApp ha sido una herramienta } \\
\text { fácil de usar }\end{array}$ & 4,3 & 4,3 \\
\hline $\begin{array}{l}\text { El grupo de WhatsApp me ha permitido } \\
\text { conocer mejor al resto de mis compañeros } \\
\text { antes del encuentro }\end{array}$ & 3,3 & 3,8 \\
\hline $\begin{array}{l}\text { Los profesores del topic me han servido } \\
\text { de guía en mi trabajo individual }\end{array}$ & 4,1 & 4,3 \\
\hline $\begin{array}{l}\text { Recomendaría el uso de WhatsApp en el } \\
\text { próximo encuentro }\end{array}$ & 4,6 & 4,7 \\
\hline $\begin{array}{l}\text { En general estoy satisfecho con el } \\
\text { programa intensivo }\end{array}$ & 4,6 & 4,4 \\
\hline
\end{tabular}

Nota: siendo 1 muy en desacuerdo y 5 totalmente de acuerdo.

Desde esta perspectiva el programa constituye una magnífica herramienta para aumentar las competencias de los estudiantes y hacerles partícipes de lo que se planteaba en un principio: convertir el aprender en una experiencia global. Destaca que los participantes están muy satisfechos tanto en lo que se refiere al programa en sí, como a la proyección profesional que perciben que les ofrece. Este hecho demuestra que se cumple el principal objetivo de preparar a los estudiantes para su inmediata incorporación al mundo laboral.

De hecho, la mayor parte de los comentarios presentados por los participantes se dirigen en este sentido. A continuación, presentamos algunos de los mismos en inglés (en el idioma original):

- Academically and professionally, it is a very rewarding programme as the topics discussed are cutting-edge and we get the chance to gain more expertise on alternative financing, ratings, very nice!!

- Great experience! Good cultural and academic programme for future years.

- It was a very beautiful experience where you learn a lot about different topics.

- It was a great experience for my future because I would like to work in an international company; cooperation is also very important for all aspects of life.

- You learn a lot from the way of working of the other (foreign) students and their culture. You also learn a lot about the city and university itself. You practice presenting in English and you talk English most of the time so this is very good for your own language skills. I would recommend it to other students!

- I had a lovely time in Zaragoza. The experience of working with other cultures has given me much knowledge how to work in an intercultural environment.

- Fun. Learning about other countries, skills from other students and how they work.

- It has been a great experience, met new people and learned a lot!

- It has been such a great experience in which I have worked with people from other countries. It's the first time Zaragoza University participates in this programme and I hope we will participate again.

- My experience along this intensive programme week was great. It allowed me to learn about the future of banking. It also was a great experience working with other cultures.

- I liked it. It helped me to understand the other cultures better.

Por tanto, este programa intensivo permite, a través de la realización de un trabajo de investigación y gracias a la cooperación internacional, que los estudiantes desarrollen las competencias que les servirán el día de mañana para un mejor desenvolvimiento profesional.

\section{CONCLUSIONES}

En el diseño de este programa intensivo y su relación con la elaboración del trabajo de fin de grado siempre se ha tenido presente la formación integral de los estudiantes, no sólo en conocimientos, sino también en otras competencias. Existe una 
clara identificación entre esta propuesta y la Estrategia Universidad puesta en marcha en España, al considerarse que la Universidad es un servicio público que tiene por objeto contribuir a la modernización de la sociedad mediante la introducción de nuevas ideas y el fomento del espíritu crítico de los estudiantes (European Commission, 2018, p.22).

Constituye un ejemplo de fomento del trabajo autónomo y cooperativo en un entorno multicultural, que permite el desarrollo de las principales competencias demandadas en el ámbito laboral. De hecho, los participantes así lo ponen de manifiesto en las encuestas que realizan al finalizar el encuentro.

Promueve además las relaciones entre universidades europeas, tanto entre estudiantes como entre docentes e investigadores. Permite el intercambio de ideas $y$ de experiencias docentes y además no es un programa estático ya que cada año, al finalizar el encuentro, se proponen mejoras para el siguiente encuentro, con el fin de profundizar en una mejor consecución de los objetivos que persigue el programa.

Por todo ello sería recomendable extender esta experiencia a otras facultades $\mathrm{y}$ universidades $\mathrm{y}$ poder ofrecer esta experiencia a nuestros estudiantes para convertirlos no sólo en mejores profesionales, sino también en mejores personas.

\section{AgRADECIMIENTOS}

Este programa no podría haberse llevado a cabo sin el liderazgo y apoyo de Petra Hogendoorn-Schweighofer y Tjerk Busstra de Inholland University of Applied Sciences (Países Bajos) que hace 7 años contactaron con el resto de Universidades para ponerlo en marcha. El éxito del último encuentro en Zaragoza ha sido posible gracias no sólo a los estudiantes que participaron y trabajaron muy duro tanto en los meses previos como durante la semana del encuentro, sino también a los profesores que les guiaron a lo largo del mismo. Es por ello por lo que queremos agradecer a los profesores Petra Hogendoorn-Schweighofer y Ruth Romijn (Inholland University of Applied Sciences), Jana Kotěšovcová, Josef Budik y Jan Mertl (University of Finance and Administration in Prague), Luc Salemans y Twan Franken (Inholland University of Applied Sciences), Jose M. Moneva, Ana Yetano e Ignacio Moralejo Menéndez (Universidad de Zaragoza), Ricardo Palomo Zurdo (Universidad CEU San Pablo) y Els Anrijs y Sven d'Hondt (University College Ghent), su participación en el VI Programa Intensivo que tuvo lugar en Zaragoza entre el 7 y el 13 de abril de 2019.

\section{REFERENCIAS}

Cátedra UNESCO (9 de octubre de 1998). Declaración Mundial sobre la educación superior en el siglo XXI: visión y acción [página web]. Recuperado de http://www.unesco.org/education/educprog/wche/declara tion_spa.htm

CEDEFOP (2014). Centro Europeo para el Desarrollo de la Formación Profesional: Desajuste de competencias: más de lo que parece a simple vista [nota informativa] Recuperado http://www.cedefop.europa.eu/files/9087_es.pdf
European Commission (2018). Promoting the relevance of higher education. Luxemburgo. Recuperado de https://publications.europa.eu/en/publication-detail//publication/59d3a999-84b9-11e8-ac6a-01aa75ed71a1

Fernández Batanero, J.M. (2004). “La transversalidad curricular en el contexto universitario: un puente entre aprendizaje académico y el natural". Revista Fuentes, 5. Recuperado https://revistascientificas.us.es/index.php/fuentes/article/ view/2403

Martínez Clares, P. y González Morga, N. (2019). "El dominio de competencia transversales en Educación Superior en diferentes contextos formativos". Educação e Pesquisa, v.45, pp. 1-23. Recuperado de http://www.scielo.br/pdf/ep/v45/1517-9702-ep-45e188436.pdf

OIT (2017). Global employment trends for youth 2017 [documento online]. Recuperado de https://www.ilo.org/wcmsp5/groups/public/---dgreports/--dcomm/---

publ/documents/publication/wcms_598669.pdf 